University of Wollongong

Research Online

Australian Institute for Innovative Materials -

Papers

Australian Institute for Innovative Materials

$1-1-2007$

Room temperature magnetic-field manipulation of electrical polarization in multiferroic thin film composite BiFeO3/La2/3Ca1/3MnO3

Zhenxiang Cheng

University of Wollongong, cheng@uow.edu.au

Xiaolin Wang

University of Wollongong, xiaolin@uow.edu.au

Follow this and additional works at: https://ro.uow.edu.au/aiimpapers

Part of the Engineering Commons, and the Physical Sciences and Mathematics Commons

Research Online is the open access institutional repository for the University of Wollongong. For further information contact the UOW Library: research-pubs@uow.edu.au 


\title{
Room temperature magnetic-field manipulation of electrical polarization in multiferroic thin film composite BiFeO3/La2/3Ca1/3MnO3
}

\author{
Abstract \\ The electrical polarization in an epitaxially $\mathrm{BiFeO}_{3}$ film grown on $\mathrm{La}_{2} / 3 \mathrm{Ca}_{1} / 3 \mathrm{MnO}_{3} / \mathrm{SrTiO}_{3}$ is observed to \\ be enhanced greatly by a magnetic field at room temperature. The simultaneous ferromagnetic order and \\ ferroelectric polarization shown by the $\mathrm{BiFeO}_{3}$ film causes the strong coupling of the magnetic and \\ ferroelectric domains in the $\mathrm{BiFeO}_{3}$ films. It was proposed that the activation energy for the electrical \\ polarization domains switching is reduced by the application of a magnetic field. As a result, the electrical \\ polarization that can be switched by an electrical field is increased by the magnetic field.

\section{Keywords} \\ Room, temperature, magnetic, field, manipulation, electrical, polarization, multiferroic, thin, film, \\ composite, BiFeO3, La2, 3Ca1, 3MnO3 \\ Disciplines \\ Engineering | Physical Sciences and Mathematics

\section{Publication Details} \\ Cheng, Z \& Wang, X (2007), Room temperature magnetic-field manipulation of electrical polarization in \\ multiferroic thin film composite BiFeO3/La2/3Ca1/3MnO3, Physical Review B (Condensed Matter and \\ Materials Physics), 75(17), pp. 172406-1-172406-4.
}




\title{
Room temperature magnetic-field manipulation of electrical polarization in multiferroic thin film composite $\mathrm{BiFeO}_{3} / \mathrm{La}_{2 / 3} \mathrm{Ca}_{1 / 3} \mathrm{MnO}_{3}$
}

\author{
Zhenxiang Cheng and Xiaolin Wang* \\ Institute for Superconducting and Electronic Materials, University of Wollongong, NSW 2522, Australia \\ (Received 19 December 2006; revised manuscript received 3 February 2007; published 30 May 2007)
}

\begin{abstract}
The electrical polarization in an epitaxially $\mathrm{BiFeO}_{3}$ film grown on $\mathrm{La}_{2 / 3} \mathrm{Ca}_{1 / 3} \mathrm{MnO}_{3} / \mathrm{SrTiO}_{3}$ is observed to be enhanced greatly by a magnetic field at room temperature. The simultaneous ferromagnetic order and ferroelectric polarization shown by the $\mathrm{BiFeO}_{3}$ film causes the strong coupling of the magnetic and ferroelectric domains in the $\mathrm{BiFeO}_{3}$ films. It was proposed that the activation energy for the electrical polarization domains switching is reduced by the application of a magnetic field. As a result, the electrical polarization that can be switched by an electrical field is increased by the magnetic field.
\end{abstract}

DOI: 10.1103/PhysRevB.75.172406

PACS number(s): 75.70.Cn, 75.80.+q

Increasing attention has been recently focused on the multiferroic materials, which exhibit simultaneous ferroelectric and ferromagnetic behaviors, because of both their intriguing physical origin and their great potential for multifunctional applications, for instance, in controlling the magnetic order parameter via electric field in a switchable manner, and vice versa. ${ }^{1-5}$ Several composite materials, consisting of separate piezoelectric and magnetic phases, have been reported to show magnetoelectric coupling at room temperature. ${ }^{6-8}$ However, room temperature single phase multiferroic materials are very limited in number, due to the chemical incompatibility between magnetism and conventional ferroelectricity. ${ }^{9,10}$ Among the few room-temperature singlephase multiferroic materials reported so far, $\mathrm{BiFeO}_{3}(\mathrm{BF})$ has the greatest potential for practical application. This is because $\mathrm{BiFeO}_{3}$ is the only known perovskite oxide that exhibits both $G$-type antiferromagnetism (weak magnetism from canted spins or parasitic ferromagnetism such as $\alpha-\mathrm{Fe}_{2} \mathrm{O}_{3}$ ) and ferroelectricity at room temperature (with $T_{N} \sim 643 \mathrm{~K}$ and $\left.T_{C} \sim 1103 \mathrm{~K}\right) .{ }^{11-14}$ The polarization and magnetization in $\mathrm{BiFeO}_{3}$ single crystal are quite small, which seems to be a strong obstacle to practical application. However, recent studies on $\mathrm{BiFeO}_{3}$ have confirmed the existence of a large ferroelectric polarization, as well as a large magnetization moment, in a strained structure formed through epitaxial growth on $\mathrm{SrRuO}_{3}$ buffered $\mathrm{SrTiO}_{3}$ (STO) substrate. ${ }^{15}$ This result has also been confirmed by theoretical predictions in terms of first-principles calculations. ${ }^{10}$ Another obstacle to the practical application of $\mathrm{BiFeO}_{3}$ is that this compound has a serious electrical leakage problem, most likely as a result of sample defects and nonstoichiometry. However, this problem could be solved by an improved fabrication process for thin films, for example, by reduced oxygen pressure, and composition modification in the ceramics. ${ }^{16-19}$ Besides all the achievements that have occurred in terms of materials processing techniques, another important achievement is the direct observation of the electrical control of antiferromagnetic domains in epitaxially grown $\mathrm{BiFeO}_{3}$ films on STO at room temperature. ${ }^{20-22}$ Using piezoforce microscopy and $\mathrm{x}$-ray photoemission electron microscopy, high-resolution images of both antiferromagnetic and ferroelectric domain structures of (001)-oriented $\mathrm{BiFeO}_{3}$ films were detected. ${ }^{20} \mathrm{~A}$ clear correlation was observed between the ferroelectric and antiferromagnetic domains, indicating a strong coupling between the two types of order. Thus, antiferromagnetic domain switching induced by ferroelectric polarization switching has been observed. However, the reverse phenomenon, that is, ferroelectric polarization domain switching by a magnetic field, has not been reported so far for $\mathrm{BiFeO}_{3}$. In this paper, we present for the first time magnetic field assisted electrical polarization in $\mathrm{BiFeO}_{3}$ thin film.

The thin film samples in this work were deposited using a pulsed laser deposition system (PLD). Third harmonic generation of a Nd:YAG laser with a wavelength of $355 \mathrm{~nm}$ and a repetition rate of $10 \mathrm{~Hz}$ was used. $\mathrm{BiFeO}_{3}$ ceramics with $5 \%$ excess bismuth and stoichiometric $\mathrm{La}_{2 / 3} \mathrm{Ca}_{1 / 3} \mathrm{MnO}_{3}$ (LCMO) ceramics, synthesized by solid state reaction were used as targets. A LCMO layer was deposited on (100) oriented $\mathrm{SrTiO}_{3}$ (STO) single crystal substrate at a temperature of $720{ }^{\circ} \mathrm{C}$ with a dynamic oxygen pressure of 200 mtorr. After the deposited film was cooled down to room temperature and reheated again, a BF layer was then deposited on the LCMO film at $600{ }^{\circ} \mathrm{C}$ at a dynamic oxygen pressure of 50 mtorr. After deposition, the double layer film was cooled down to room temperature in a half hour. The phase and structure of the films was determined by x-ray diffraction (XRD) using $\mathrm{Cu} K \alpha$ radiation. The surface morphology of the as-deposited films was scanned by an atomic force microscope (AFM) in tapping mode. A Pt upper electrode with an area of $0.00785 \mathrm{~mm}^{2}$ was deposited by magnetron sputtering through a metal shadow mask. The thickness of the films was obtained through observation of the cross section of the film under a high resolution scanning electron microscope (SEM); the BF and LCMO layers are each about $300 \mathrm{~nm}$. Magnetic properties of the sample were investigated on a Quantum Design magnetic properties measurement system (MPMS). The ferroelectric properties were measured by an aixACCT EASY CHECH 300 ferroelectric tester. During the measurement of the polarization-electrical field loop (P-E loop), a static magnetic field of 1000 Oe was applied along the film normal.

The $\theta-2 \theta$ XRD pattern of the double layered thin film is shown in Fig. 1. Epitaxial growth of LCMO along its (010) direction on (001) STO substrate was confirmed, since no trace of diffraction from other directions was observed. The $\mathrm{BF}$ layer on LCMO shows a tetrahedral structure, which is 


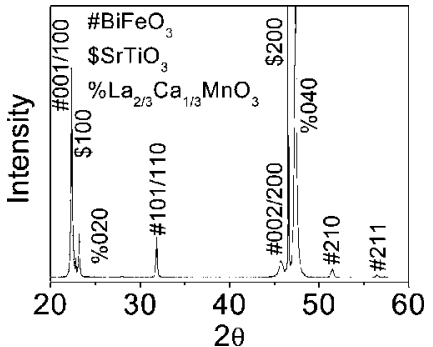

FIG. 1. The $\theta-2 \theta$ x-ray diffraction pattern of the $\mathrm{BiFeO}_{3}$ $/ \mathrm{La}_{2 / 3} \mathrm{Ca}_{1 / 3} \mathrm{MnO}_{3}$ double layer thin film on $\mathrm{SrTiO}_{3}$, indicating epitaxial growth of the $\mathrm{La}_{2 / 3} \mathrm{Ca}_{1 / 3} \mathrm{MnO}_{3}$ film along its (010) direction on (100) $\mathrm{SrTiO}_{3}$, and preferred growth of the tetragonal $\mathrm{BiFeO}_{3}$ film along its (001) direction.

different from the bulk rhombohedral structure. To compare with the diffraction pattern of the ceramic sample, preferred growth of $\mathrm{BF}$ along its (001) direction was observed on the LCMO buffer layer. The growth habit of the tetragonal BF and LCMO can be easily explained by the diffraction positions of the STO (100), LCMO (010), and BF (001) peaks, and the epitaxial growth relationship of the faces mentioned above was obtained.

A typical surface morphology of the as-grown BF films is shown in Fig. 2. It was found that the maximum grain size can reach up to $300 \mathrm{~nm}$, while the average grain size is less than $200 \mathrm{~nm}$. The surface is quite smooth with a root-meansquare roughness of less than $8 \mathrm{~nm}$.

Figure 3 shows magnetization hysteresis loops (M-H loops) of the BF/LCMO thin film composite measured at $300 \mathrm{~K}$ and $10 \mathrm{~K}$. The composite is ferromagnetic at both room temperature and $10 \mathrm{~K}$. It is known that $\mathrm{BiFeO}_{3}$ is antiferromagnetic below its Néel temperature of $643 \mathrm{~K}$, and its weak ferromagnetism is from the breaking of the long spiral structure of the tilted antiferromagnetic arrangement of spins

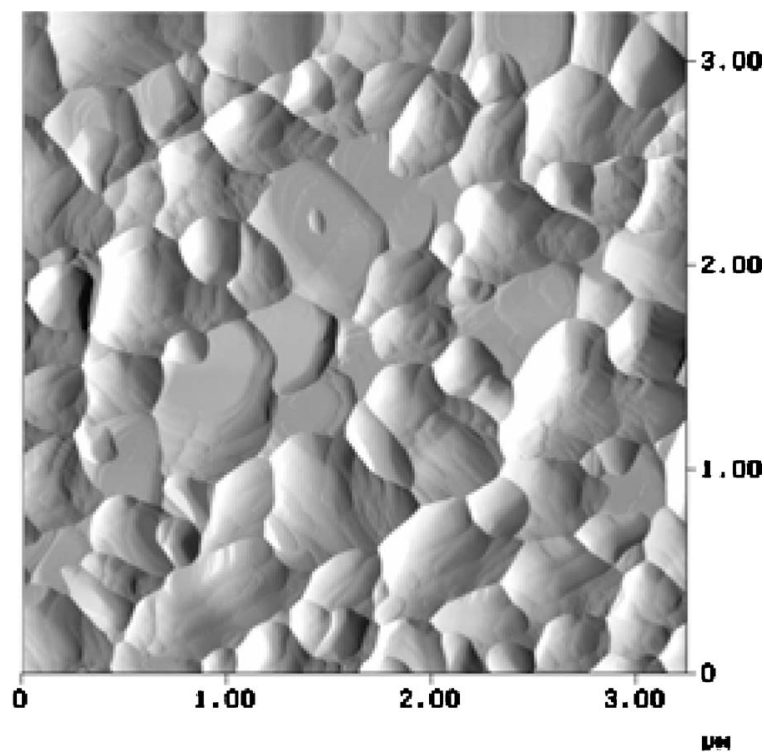

FIG. 2. Surface morphology of $\mathrm{BiFeO}_{3}$ film on $\mathrm{La}_{2 / 3} \mathrm{Ca}_{1 / 3}$ $\mathrm{MnO}_{3} / \mathrm{SrTiO}_{3}$ in a $3.2 \times 3.2 \mu \mathrm{m}^{2}$ area scanned by atomic force microscope (AFM) in tapping mode.

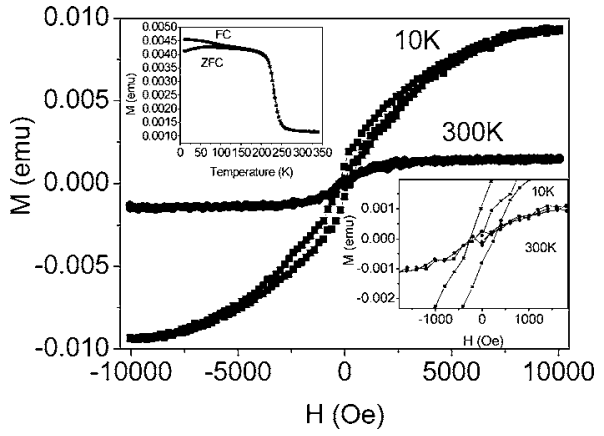

FIG. 3. Magnetization hysteresis loops of $\mathrm{BiFeO}_{3}$ $/ \mathrm{La}_{2 / 3} \mathrm{Ca}_{1 / 3} \mathrm{MnO}_{3}$ double layer composite measured at $10 \mathrm{~K}$ and $300 \mathrm{~K}$. The inset is the FC-ZFC magnetization curve at 1000 Oe.

in the thin film. The LCMO is paramagnetic at room temperature, which is far above its ferromagnetic $T_{C}$ of $250 \mathrm{~K}$. This is also proved by the field-cooled and zero field-cooled (FC-ZFC) measurements of the magnetic susceptibility, shown in the inset of the figure. So the ferromagnetism shown by the thin film composite at room temperature is from the BF layer, while both the LCMO and the BF contribute to the magnetization at $10 \mathrm{~K}$. This indicates that both component layers in the BF/LCMO composite maintain their own ferromagnetism, revealing a good interfacial compatibility of the two materials.

Figure 4 shows electrical polarization-electrical field (P-E) hysteresis loops of a Pt/BF/LCMO capacitor measured at different maximum applied voltages. The remanent polarization $\left(2 P_{r}\right)$ values of the capacitor measured at maximum voltages of $10 \mathrm{~V}$ and $14 \mathrm{~V}$ are 5.4 and $7.3 \mu \mathrm{C} / \mathrm{cm}^{2}$, respectively, which is below the value after the sample is completely switched. However, the complete switching of the polarization was hindered by electrical leakage, which might be caused by oxygen vacancy ionic conductivity. After a 1000 Oe magnetic field was applied, the P-E loops were measured again.

A comparison of the polarization measured with and without magnetic field applied is plotted in Fig. 5. It was found that the loops were tilted up by the magnetic field, revealing an increase in the saturated polarization $\left(P_{s}\right.$, with the $P$ at the maximum voltage here denoted by $P_{s}$, although the polarization is not completely switched). For example, the $P_{s}$ values

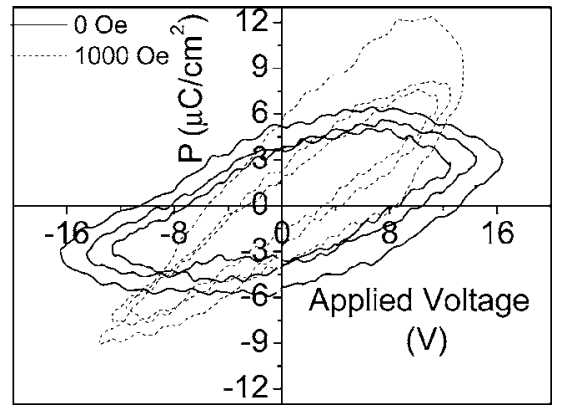

FIG. 4. Polarization-electrical field hysteresis loops measured at different applied voltages and zero magnetic field (solid line) and at a magnetic field of 1000 Oe (dotted line) ( $H$ is along the film surface normal). 


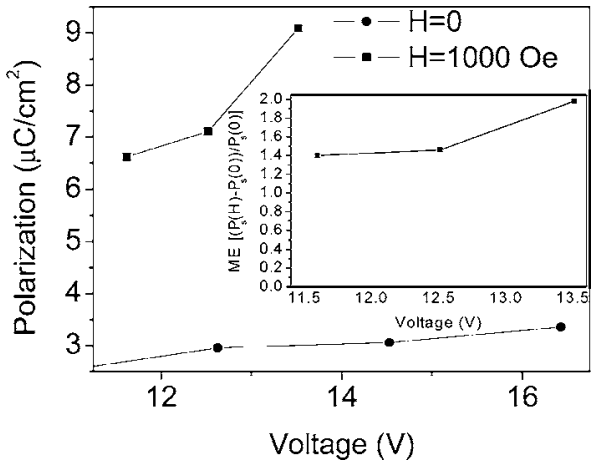

FIG. 5. Comparison of polarization of $\mathrm{Pt} / \mathrm{BiFeO}_{3}$ $/ \mathrm{La}_{2 / 3} \mathrm{Ca}_{1 / 3} \mathrm{MnO}_{3}$ capacitor measured without magnetic field applied and with a 1000 Oe magnetic field applied. Inset figure is the ME effect in terms of $\left[P_{S}(H)-P_{S}(0)\right] / P_{S}(0)$.

at a maximum applied voltage of $13 \mathrm{~V}$ are $2.6 \mu \mathrm{C} / \mathrm{cm}^{2}$ and $7.6 \mu \mathrm{C} / \mathrm{cm}^{2}$ when no magnetic field and a 1000 Oe static magnetic field were applied, respectively. An ME effect of $196 \%$ in terms of $P_{s}$ enhancement, defined as $\left[P_{s}(H)-P_{s}(0)\right] / P_{s}(0)$, was obtained at 1000 Oe magnetic field. However, it was found that $P_{r}$ remains almost unchanged under the magnetic field. It is worth indicating that the ME effect defined here should include the contribution from the possible linear and high rank ME effect due to the structure change of $\mathrm{BiFeO}_{3}$ thin film and unique polarization hysteresis loop measurement under a magnetic field. ${ }^{23}$

To elucidate the origin of the observed ME effect in our double layer thin film composite, the magnetoresistance (MR) behavior of the bottom electrode was examined. It was found that the bottom LCMO electrode has good conductivity, but no MR effect was observed at room temperature. This is also in accordance with the magnetic measurements on the sample, that is, LCMO is paramagnetic above its $T_{C}$ of $250 \mathrm{~K} \cdot{ }^{24,25}$ So, any effect from the bottom electrode could be ruled out. The origin of the ME effect in the BF/LCMO thin film composite is believed to come from the intrinsic properties of the multiferroic $\mathrm{BF}$ layer. The ME effect in $\mathrm{BF}$ has been confirmed using first-principles density functional theory calculations. ${ }^{9,10}$ The Dzyaloshinskii-Moriya interaction, leading to the canting of the magnetic moments and the appearance of macroscopic magnetization, is a result of polar distortion in BF. As a result, the magnetization is strongly coupled to the ferroelectric polarization, and the reversal of the ferroelectric polarization by an electric field will be accompanied by the reversal of the macroscopic magnetization. This is proved by the direct observation of the switching behavior of the antiferromagnetic domains in $\mathrm{BF}$ in response to an electrical field at room temperature. ${ }^{20}$ The observation also showed that antiferromagnetic domains and ferroelectric domains were present in the same domain area, which means that the vectors of the ferroelectric domains and antiferromagnetic domains are strongly coupled together no matter how the ferroelectric domains or antiferromagnetic domains are switched.
In BF thin film with a suppressed long-wavelength spiral structure, the "easy magnetization plane," where individual spin vectors fall in the $G$-type antiferromagnetic order, is perpendicular to the polarization direction. ${ }^{19}$ When a switching electrical field is applied, the switching of the ferroelectric polarization will couple to the ferroelastic strain, which will cause the reorientation of the antiferromagnetic spins according to the above relationship between the electrical polarization and antiferromagnetic vectors. However, the opposite method of control in $\mathrm{BiFeO}_{3}$, that is, electrical polarization domain switching by a magnetic field, has not been reported. Probably it is technically difficult to realize the switching of electrical polarization only by a magnetic field. Our sample reveals that the magnetic field facilitates the electrical polarization switching by an electrical field, but the electrical polarization may not be switched by such a magnetic field alone. The $\mathrm{M}-\mathrm{H}$ loops of our sample reveal the weak ferromagnetic magnetization behavior of the sample under a magnetic field of 1000 Oe (shown in the inset of Fig. 2 in the bottom-right corner). The ferromagnetic magnetization of the BF thin film is a process involving the recanting of the antiferromagnetic spins. However, the antiferromagnetic vector may not be switched by such a small magnetic field. When a switching electrical field was applied, the switching of the ferroelectric polarization will cause the reorientation of the antiferromagnetic spins. So part of the energy will be consumed by the switching of antiferromagnetic domains.

When a magnetic field is applied, it helps the recanting of the spin by facilitating the antiferromagnetic domain switching. In such a way, the activation energy for the switching of the electrical polarization domains is reduced. As a result, the electrical polarization that could be switched by an electrical field is increased by a magnetic field. Thus, the P-E loops for our BF films are tilted up in a high electrical field by a magnetic field.

In summary, we have grown BF thin film on LCMO epitaxially grown on a STO substrate. Both ferromagnetic and ferroelectric properties were demonstrated by the BF film. We observed for the first time that the electrical polarization in $\mathrm{BiFeO}_{3}$ films can be enhanced greatly by magnetic field at room temperature. Due to the fact that both magnetic and ferroelectric domains are strongly coupled together in the BF films, we proposed that the activation energy for the electrical polarization domains switching is reduced by the application of a magnetic field. As a result, the electrical polarization that can be switched by an electrical field is increased by the magnetic field. Further experimental work in the future will be focused on the temperature and magnetic field dependence of the ME effect, thus it is expected that this explanation will acquire more evidence.

This work is supported by the Australian Research Council (ARC) through Discovery projects DP0558753 and DP0665873. 
*Corresponding author. Electronic address: xiaolin@uow.edu.au

${ }^{1}$ M. Fiebig, J. Phys. D 38, R123 (2005).

${ }^{2}$ W. Prellier, M. P. Singh, and P. Murugavel, J. Phys.: Condens. Matter 17, R803 (2005).

${ }^{3}$ T. Kimura, T. Goto, H. Shintani, K. Ishizaka, T. Arima, and Y. Tokura, Nature (London) 426, 55 (2003).

${ }^{4}$ N. Hur, S. Park, P. A. Sharma, J. S. Ahn, S. Guha, and S.-W. Cheong, Nature (London) 429, 392 (2004).

${ }^{5}$ W. Eerenstein, N. D. Mathur, and J. F. Scott, Nature (London) 442, 759 (2006).

${ }^{6}$ H. Zheng, J. Wang, S. E. Lofland, Z. Ma, L. Mohaddes-Ardabili, T. Zhao, L. Salamanca-Riba, S. R. Shinde, S. B. Ogale, F. Bai, D. Viehland, Y. Jia, D. G. Schlom, M. Wuttig, A. Roytburd, and R. Ramesh, Science 303, 661 (2004).

${ }^{7}$ M. A. Zurbuchen, T. Wu, S. Saha, J. Mitchell, and S. K. Streiffer, Appl. Phys. Lett. 87, 232908 (2005).

${ }^{8}$ J. P. Zhou, H. C. He, Z. Shi, and C. W. Nan, Appl. Phys. Lett. 88, 013111 (2006).

${ }^{9}$ N. A. Hill, J. Phys. Chem. B 104, 6694 (2000).

${ }^{10}$ P. Baettig, C. Ederer, and N. A. Spaldin, Phys. Rev. B 72, 214105 (2005).

${ }^{11}$ Z. X. Cheng, X. L. Wang, C. V. Kannan, K. Ozawa, H. Kimura, T. Nishida, S. J. Zhang, and T. R. Shrout, Appl. Phys. Lett. 88, 132909 (2006).

${ }^{12}$ J. R. Cheng, S. W. Yu, J. G. Chen, Z. Y. Meng, and L. Eric Cross, Appl. Phys. Lett. 89, 122911 (2006).

${ }^{13}$ C. Ederer and N. A. Spaldin, Phys. Rev. B 71, 060401(R) (2005).

${ }^{14}$ J. Dho, X. Qi, H. Kim, J. L. MacManus-Driscoll, and M. G.
Blamire, Adv. Mater. (Weinheim, Ger.) 18, 1445 (2006).

${ }^{15}$ J. Wang, J. B. Neaton, H. Zheng, V. Nagarajan, S. B. Ogale, B. Liu, D. Viehland, V. Vaithyanathan, D. G. Schlom, M. Wuttig, and R. Ramesh, Science 299, 1719 (2003).

${ }^{16}$ X. D. Qi, J. Dho, R. Tomov, M. G. Blamire, and J. L. MacManusDriscoll, Appl. Phys. Lett. 86, 062903 (2005).

${ }^{17}$ D. H. Wang, W. C. Goh, M. Ning, and C. K. Ong, Appl. Phys. Lett. 88, 212907 (2006).

${ }^{18}$ K. Y. Yun, M. Noda, M. Okuyama, H. Saeki, H. Tabata, and K. Saito, J. Appl. Phys. 96, 3399 (2004).

${ }^{19}$ S. T. Zhang, M. H. Lu, Y. F. Chen, and N. B. Ming, Appl. Phys. Lett. 87, 262907 (2005).

${ }^{20}$ T. Zhao, A. Scholl, F. Zavaliche, K. Lee, M. Barry, A. Doran, M. P. Cruz, Y. H. Chu, C. Ederer, N. A. Spaldin, R. R. Das, D. M. Kim, S. H. Baek, C. B. Eom, and R. Ramesh, Nat. Mater. 5, 823 (2006).

${ }^{21}$ F. Zavaliche, P. Shafer, R. Ramesh, M. P. Cruz, R. R. Das, D. M. Kim, and C. B. Eom, Appl. Phys. Lett. 87, 252902 (2005).

${ }^{22}$ F. Zavaliche, R. R. Das, D. M. Kim, C. B. Eom, S. Y. Yang, P. Shafer, and R. Ramesh, Appl. Phys. Lett. 87, 182912 (2005).

${ }^{23}$ C. Tabares-Munoz, J. -P. Rivera, A. Bezinges, A. Mounnier, and H. Schmid, Jpn. J. Appl. Phys., Suppl. 24(Supplement), 1051 (1985).

${ }^{24}$ Z. X. Cheng, X. L. Wang, and S. X. Dou, J. Magn. Magn. Mater. 283, 143 (2004).

${ }^{25}$ Z. X. Cheng, H. F. Zhen, A. H. Li, X. L. Wang, and H. Kimura, J. Cryst. Growth 275, e2415 (2005). 